POLITICAS

\title{
Reducción de la violencia y la delincuencia en Bogotá, Colombia, 1994-2002
}

\author{
Hugo Acero \\ Subsecretaría de Asuntos de Convivencia y Seguridad Ciudadana, Bogotá D.C., Colombia
}

\begin{abstract}
El presente artículo tiene como objetivos mostrar con datos confiables los índices de reducción, fundamentalmente de los homicidios comunes, y presentar las políticas que se han aplicado en forma sostenida en Bogotá en los últimos ocho años, que se consideran el soporte de esta reducción.
\end{abstract}

Palabras clave: reducción homicidios, políticas, Bogotá, Colombia.

Violence and criminality decrease in Bogota, Colombia, 1994-2002

The present article shows reliable data on Bogota's homicide decreasing rates and presents the strategies applied during the past 8 years which partly explain such decrease.

Key words: decrease in homicides, strategies, Bogota, Colombia.

\section{Presentación}

En los últimos años en la capital de Colombia, Bogotá, se ha reducido significativamente la violencia homicida, además de otros delitos. Este proceso se inició en 1994, cuando las tasas de homicidio pasaron de 80 homicidios comunes por cada 100.000 hab en 1993 a 30,7 en el año 2001, es decir, una disminución de la tasa de $62 \%$ en 8 años. En términos absolutos se pasó de 4.352 homicidios comunes en 1993 a 2.012 en el año 2001 (cuadro 1).

Esta reducción de los homicidios en Bogotá, resulta más notoria si se tiene en cuenta que este delito ha aumentado en el país y en sus dos principales ciudades, Cali y Medellín (figura 1).

En Colombia, Bogotá ocupa el puesto 25 dentro de las capitales de los 32 departamentos en que esta dividido el país. A diferencia de otras capitales de las Américas, la capital de Colombia, no es la más violenta. De hecho, si se le compara con otras ciudades de las Américas, en relación con la tasa de homicidios comunes por cada 100.000 hab, Bogotá tiene una tasa inferior a Medellín, Cali,

Correspondencia:

Carrera 8 No. 10-65, telefono 3417006

Recibido: 28/10/02; aceptado: 20/11/02
Cuadro 1. Comportamiento del homicidio común, Bogotá, 1990-2001.

\begin{tabular}{lccc}
\hline Año & Población & $\begin{array}{c}\text { Homicidio } \\
\text { común }\end{array}$ & $\begin{array}{c}\text { Tasa por } \\
\mathbf{1 0 0 . 0 0 0} \text { hab }\end{array}$ \\
\hline 1991 & $5^{\prime} 172,313$ & 2.890 & 55,9 \\
1992 & $5^{\prime} 306,357$ & 3.352 & 63,2 \\
1993 & $5^{\prime} 440,401$ & 4.352 & 79,0 \\
1994 & $5^{\prime} 574,445$ & 3.885 & 69,7 \\
1995 & $5^{\prime} 708,489$ & 3.363 & 58,9 \\
1996 & $5^{\prime} ' 442,533$ & 3.303 & 56,5 \\
1997 & $5^{\prime} 976,579$ & 2.814 & 47,1 \\
1998 & $6^{\prime} 123,306$ & 2.482 & 40,5 \\
1999 & $6^{\prime} 271,260$ & 2.477 & 39,0 \\
2000 & $6^{\prime} 422,794$ & 2.238 & 34,8 \\
2001 & $6^{\prime} 553,622$ & 2.012 & 30,7 \\
\hline
\end{tabular}

Fuente: Instituto Nacional de Medicina Legal

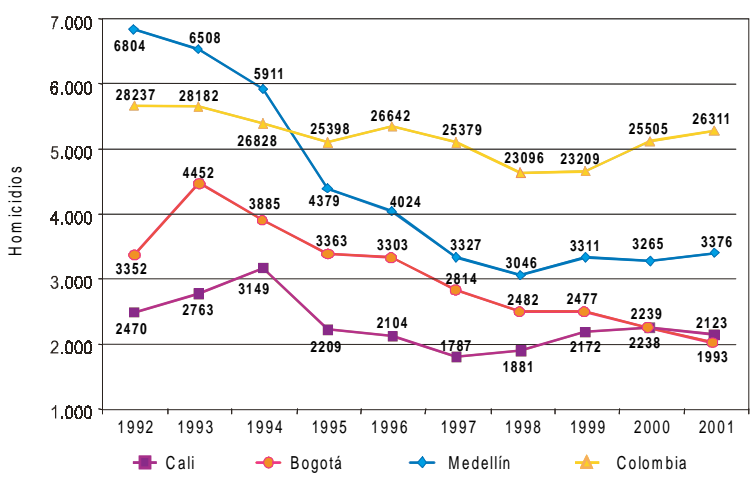

Figura 1. Homicidio común en tres ciudades de Colombia y en Colombia,1992-2001. 


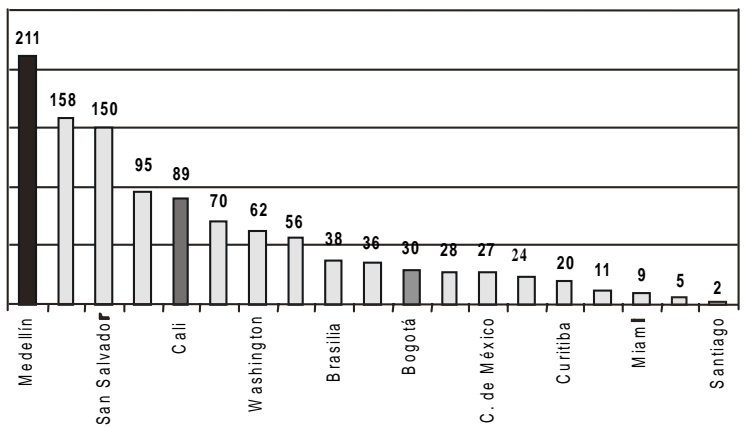

Fuente: Revista América Economía, 2000

Figura 2. Comparación tasa de homicidios por 100.000 hab en ciudades de las Américas.

Washington, Caracas, Sao Paulo, Caracas, Río de Janeiro y San Salvador, entre otras, pero superior a Lima, Ciudad de México, Curitiba, Ciudad de Panamá, Miami, Buenos Aires y Santiago de Chile (figura 2).

Además de estos logros en materia de violencia homicida, las muertes en accidentes de tránsito se redujeron en un $46 \%$ en 6 años; se pasó de 1.387 casos en 1995 a 745 en el 2001; es decir, de una tasa de 24 muertes en accidentes de tránsito por cada 100.000 hab a 11 (figura 3). En lo que hace a los otros delitos de mayor impacto ${ }^{1}$, también se redujeron desde1998; se pasó de 25.121 delitos registrados en 1997 a 17.616 en el 2001 (figura 4).

Estos logros se atribuyen a la aplicación de una política integral de seguridad y convivencia que

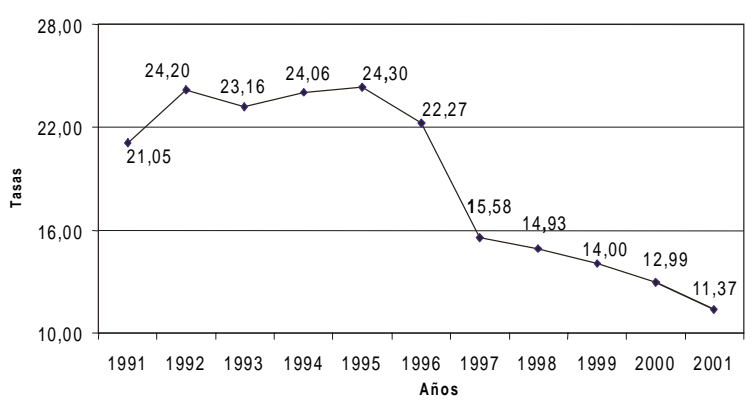

Fuente: Instituto Nacional de Medicina Legal y Ciencias forenses

Figura 3. Muertes en accidentes de tránsito en Bogotá, D.C., tasa por 100.000 hab, 1991-2001.

\footnotetext{
${ }^{1}$ La policía clasifica como delitos de mayor impacto los hurtos de autos, motos y residencias, el atraco callejero, el hurto bancario y de establecimientos comerciales y las lesiones personales.
}

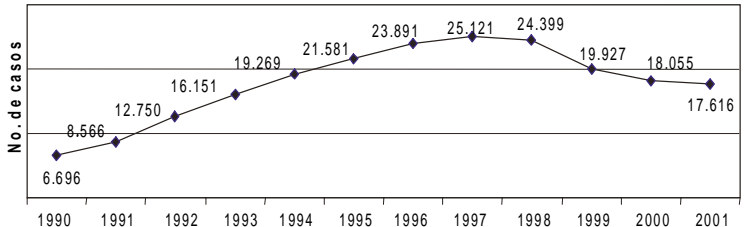

Fuente: Policía Metropolitana de Bogotá.

Figura 4. Delitos de impacto social en Bogotá.

la ciudad ha venido ejecutando desde 1995, la cual incluye diferentes proyectos y programas que pasan por el fortalecimiento de las instituciones responsables de garantizar la seguridad y la convivencia de los ciudadanos, como son la policía y el sistema de justicia, hasta el desarrollo de una serie de políticas públicas orientadas a la prevención de la violencia y el delito, las cuales se expondrán más adelante.

\section{Intervenciones a la dismimución de la violencia y la delincuencia}

¿Cómo explicar la constante reducción de la violencia homicida registrada que viene observándose en Bogotá desde hace 8 años? ¿Cuál es el peso atribuible a cada factor dentro de los diversos proyectos que se han puesto en marcha en ese periodo? A estas preguntas y otras similares no se ha respondido adecuadamente. Aunque pocos estudios se han acercado a responderlas, es posible hacerlo. De hecho, un estudio sobre los efectos de las medidas de prohibición del porte de armas determinó que esas medidas aplicadas en Cali y Bogotá explicaron la reducción del $13 \%$ de los homicidios (1).

Otras teorías se mencionan a continuación, algunas vienen de la academia y otras de las instituciones.

\section{Una tendencia nacional}

Se ha postulado que en el caso de Bogotá la disminución de la violencia en los últimos años responde a una tendencia 'normal' que aplica a todo el país e, incluso, a la mayoría de ciudades y países del mundo. Así lo manifiesta un estudio presentado por la Universidad del Rosario (2),según el cual la tasa de homicidios de Bogotá sube siguiendo la del país hasta 1993, año en que, también con similar comportamiento al del país comienza a descender. El hecho de que sea 
similar al país significa que es el resultado de una mejora en el sistema de justicia y policía de toda la Nación ${ }^{2}$.

Sin embargo, las cifras reflejan una realidad diferente como lo muestra la figura 1. Mientras Bogotá mantiene la tendencia a la baja desde 1994, el país, Medellín y Cali volvieron a aumentar sus índices de violencia entre 1997 y 1998.

Por otro lado, existen zonas del país que mantienen una tendencia al aumento del homicidio, como es el caso de los departamentos de Bolívar, que pasó de 240 homicidios registrados en 1990 a 453 en 2001, 213 casos más; Norte de Santander, de 696 en 1990 a 928 en 2001, 232 casos más; Santander, de 801 en 1990 a 1.090 en 2001, 289 casos más; Tolima, de 463 en 1990 a 553 en 2001, 90 casos más; Huila, de 330 en 1990 a 381 en 2001, 51 casos más ${ }^{3}$.

\section{Violencia, vigilancia y control}

Por su parte, Mauricio Rubio apunta otra explicación respecto a la reducción de la violencia y la delincuencia: la relación entre violencia, vigilancia y control. Esta hipótesis se basa en la teoría económica del crimen, conocida como la hipótesis de la disuasión, o sea, la consideración del efecto que tienen las sanciones del Estado sobre la incidencia de conductas criminales. La teoría señala que la decisión de cometer un crimen o acto violento es consecuencia directa del supuesto de racionalidad de los delincuentes para quienes la persecución y la sanción constituyen una especie de costo, un precio de sus actividades ilegales. Al incrementarse el control, este precio actuaría como un incentivo tendiente a disminuir la incidencia de tales conductas ${ }^{4}$.

\footnotetext{
2 Al respecto, según datos de Medicina Legal, entre 1993 y 2000 el porcentaje de participación de los homicidios de la ciudad con relación al total nacional disminuyó del 15,8 al 9,6 es decir, se redujo en un $40 \%$, aproximadamente; por tanto, esta afirmación pierde consistencia.

3 Fuente: para 1990 DIJIN - CIC, para 2001 Instituto Nacional de Medicina Legal. Como vemos, la tesis tampoco se puede soportar con las cifras departamentales.

${ }^{4}$ Alcaldía Mayor de Bogotá, Secretaría de Gobierno, Universidad de Los Andes. 2000. "Caracterización de la Violencia Homicida en Bogotá", Estudio interpretativo de la violencia homicida en Bogotá. Documento sin editar.
}

Esta hipótesis en parte es cierta, si se tiene en cuenta que la operatividad de la policía, frente a la comisión de delitos, ha mejorado ${ }^{5}$. Sin embargo hay que reconocer la importancia de las acciones preventivas del plan integral de seguridad y convivencia de Bogotá que han tenido incidencia en la reducción de la violencia homicida, por ejemplo, las políticas de desarme y de cierre de establecimientos nocturnos donde se expende licor a la 1:00 a.m. que, según en estudio de la Universidad de los Andes, anteriormente mencionado, representaron una disminución del $22 \%$.

\section{Causas objetivas}

Otros afirman, desde la perspectiva de causas de la violencia y la delincuencia, que hay unas 'causas objetivas' que las explican y éstas se relacionan con factores sociales y económicos tales como la pobreza, la desigualdad o injusticia social, el desempleo y la marginalidad producto de los excesivos flujos migratorios del campo hacia la ciudad ${ }^{6}$. Por tanto, si se intervienen esos factores se espera reducir los hechos de violencia y delincuencia.

El caso de Bogotá no se ajustaría a este tipo de relaciones causales puesto que en los últimos años el desempleo, la población desplazada y los niveles de pobreza en el país en general y en Bogotá en particular han aumentado, mientras que las cifras de violencia y delincuencia van en dirección opuesta.

\footnotetext{
5 Con menos de los 10.500 que la ciudad tiene desde 1994 se logró la reducción de los índices de violencia y delincuencia. Por otra parte, la percepción de la institución por parte de la ciudadanía ha mejorado sustancialmente. De ser considerada una de las instituciones más corruptas a comienzos de los años 90 del siglo pasado, la última encuesta de Napoleón Franco (octubre 2002) la ubica entre las cinco primeras instituciones del Distrito Capital.

6 No obstante, la relación violencia-condiciones sociales ha sido fuertemente discutida. Al respecto, ver Alcaldía Mayor de Bogotá, Secretaría de Gobierno, Universidad de Los Andes, "Caracterización de la violencia homicida en Bogotá", Isaac Beltrán, Ana María Fernandez, Fernando Gaitán, 2000 (2) y "Política de seguridad y gasto en Bogotá", Misión de la reforma institucional del Distrito, Universidad del Rosario.
} 


\section{Los ciclos de la violencia}

En el caso de la violencia, Gaitán (3) muestra cómo no es posible hablar de violencia cíclica, es decir transiciones entre "alta violencia, violencia, baja violencia, normal y, de ahí, comenzar a ascender", aunque desarrolla un argumento en el cual en Colombia ha habido periodos de alta y baja intensidad de violencia. EI comportamiento de dichos periodos estarían asociados con intervenciones institucionales tendientes a presionar una disminución de la violencia en momentos álgidos.

Con este argumento, según el cual existen períodos en el país en los que la violencia aumenta y otros en los que disminuye, se arguye que al observar la tendencia de los homicidios se destacan los picos y ondulaciones que se inician con la Guerra de los Mil Días, la violencia partidista de los años 40 y 50 , la violencia asociada con el tráfico de drogas de los años 70 y 80 y el escalonamiento de las actividades de los grupos armados en el campo y zonas urbanas. Estos dos últimos hechos explicarían la actual ola de violencia social que ha generado entre 25.000 y 27.000 homicidios anuales en la última década.

Para el caso de Bogotá, la curva de homicidios muestra el mismo patrón que el resto del país hasta 1993, como se ve en la figura 5, cuando la curva de Bogotá comenzó un proceso de reducción, como se vio en la figura 1 , contraria a la del país que comenzó a aumentar desde 1997. Es decir, no hay evidencia histórica que muestre periodos anteriores de altos y bajos niveles de violencia, por los cuales se pueda considerar que

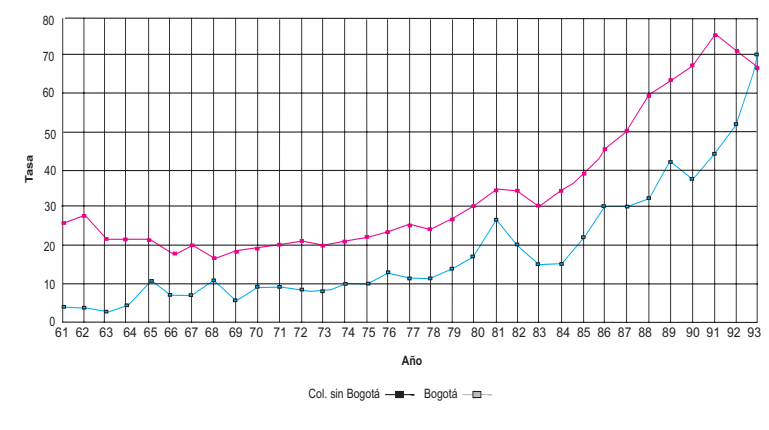

Tomado de: Cámara de Comercio de Bogotá (4)

Figura 5. Comportamiento del homicidio. Tasa por 100.000 habitantes. la actual reducción de homicidios en Bogotá corresponda a un nuevo ciclo hacia la baja.

\section{Hora zanahoria y desarme $^{7}$}

Sobre los efectos de las medidas conocidas como la 'hora zanahoria' y el desarme, el estudio realizado para la Universidad de Los Andes sobre el tema de violencia homicida estimó que "... tomando en cuenta que desde que se adoptó la hora zanahoria (1:00 a.m.) en diciembre de 1995 hasta finales de 1999, el número promedio de homicidios en Bogotá ha sido de 56 por semana, la magnitud del efecto de esta intervención en la reducción de la tendencia de los homicidios sería del orden del $8 \%$. En el caso del control de armas durante los fines de semana, se presentaron 61 homicidios en promedio semanal desde su primer período de vigencia a principios de 1994 hasta finales de 1999; así, la magnitud del efecto de esta medida no superaría el $14 \%$ de la reducción en la tendencia de los homicidios en la ciudad" ${ }^{\text {. }}$.

Estas dos medidas en conjunto, de acuerdo con el mencionado estudio, contribuyeron en un $22 \%$ en las reducciones de los homicidios de la ciudad entre 1995 y 1999 . Pero este dato debe ser revisado con cuidado, no sólo porque no explica la variación total, sino porque es necesario estudiar otras explicaciones que correspondan de manera más clara con demostraciones científicas.

\section{Causas no explicables o multicausalidad}

Otros consideran que la reducción de la violencia y de la delincuencia en Bogotá se explica por la variedad e integralidad de las diferentes acciones que se empezaron a aplicar desde 1995.

Se parte de reconocer que la violencia y la delincuencia es multicausal, tal y como ha sido sustentado por diversos tratadistas y estudiosos del tema. Por ello, es necesario estudiar el impacto de un número importante de proyectos orientados

\footnotetext{
7 Se denominó 'hora zanahoria' al cierre de los establecimientos nocturnos que expenden licor a la 1:00 a.m.

8 Alcaldía Mayor de Bogotá, Secretaría de Gobierno, Universidad de Los Andes. 2000. "Caracterización de la violencia homicida en Bogotá, efectos de los controles al consumo de alcohol y al porte de armas de fuego en la violencia homicida". Documento sin editar.
} 
a mejorar la convivencia y seguridad, definidos y financiados por las últimas tres administraciones distritales con la formulación de una política integral de seguridad y convivencia en 1995. Desde ese año se dio un paso adelante para reconocer que la seguridad no es de la exclusiva responsabilidad de las autoridades armadas y de justicia, sino que debe abordarse con las autoridades administrativas, a través de una política pública clara que de manera sostenida, como política de Estado y no del gobernante de turno, planifique y oriente a la creación de ciudadanía y mejoría de los índices de convivencia y seguridad humana.

\section{Programas adelantados}

\section{Mejoramiento del servicio de la Policía}

Con el objetivo de mejorar la acción de la Policía Metropolitana ${ }^{9}$ frente a la prevención de la violencia y la delincuencia, las administraciones distritales, en los últimos años, aumentaron significativamente el presupuesto para fortalecer esta institución. Se pasó de \$12.157 millones que invirtió la administración de Jaime Castro (19941995) a $\$ 49.533$ millones del primer gobierno de Antanas Mockus (1997-1997); la administración de Enrique Peñalosa ejecutó \$116.107 millones (1998-2000) y, en la actualidad, Mockus ha presupuestado una ejecución de $\$ 121.742$ millones (2001-2003), así:

$\begin{array}{lll}\text { Año } & \text { Presupuesto } & \text { Ejecutado } \\ 2001 & \$ 26.459 & \$ 26.266 \\ 2002 & \$ 40.810 & \$ 18.792^{*} \\ 2003 & \$ 54.666 & -------\end{array}$

* a 31 de octubre de 2002

Con estos recursos, y en coordinación con los distintos comandantes de la Policía Metropolitana de Bogotá, se han ejecutado las siguientes estrategias:

9 Colombia tiene una sola policía que es la Policía Nacional, la cual depende directamente del despacho del Ministro de Defensa. La Policía Metropolitana es una unidad de la institución nacional. Desde 1994 la ciudad cuenta con algo menos de 10.500 agentes y aunque los distintos gobiernos distritales han demandado el aumento del pie de fuerza, sin lograr este objetivo, cabe resaltar cómo la estrategia de seguridad y convivencia no se sustentó en el aumento de la policía. a) Con el fin de fortalecer técnicamente a la Policía, desde finales de 1994, se inició un proceso de modernización del Centro Automático de Despacho, CAD ${ }^{10}$, que se tradujo en una disminución sustancial en los tiempos de respuesta a los requerimientos ciudadanos; se pasó de 20 minutos promedio de respuesta a 5 . Por otro lado, desde 1995 se comenzó la modernización y ampliación del parque automotor, con lo cual no sólo se hizo la reposición de los que se encontraban fuera de servicio, sino que prácticamente se duplicó el número de patrullas y motos, lo que significó mayor movilidad. En 1995, la Policía contaba con 286 carros y 172 motos; hoy, la Policía cuenta con 474 vehículos y 683 motos, lo que le permite tener mayor movilidad.

b) Con la perspectiva de brindar un servicio con calidad, en 1998 se reorientó el programa de los Centros de Atención Inmediata, CAI, los cuales se reubicaron en sitios estratégicos de la ciudad, con base en el análisis pormenorizado de la actividad delictiva por sectores. Al mismo tiempo, se establecieron nuevas jurisdicciones, evitando así la superposición de servicios o modalidades de vigilancia, se disminuyó su número y se aumentó su personal y los medios de apoyo. Además de los CAI, se construyeron y se mejoraron la mayoría de las estaciones de policía de las localidades, hoy sólo faltan dos estaciones (Puente Aranda y Ciudad Bolívar) que están proyectadas en el nuevo Plan de Desarrollo junto con nuevos CAI.

c) Las acciones no se limitaron a su fortalecimiento material, sino también a mejorar el talento humano. Desde 1996, se destinaron recursos para coadyuvar a la capacitación y actualización de, aproximadamente, 8.000 oficiales, suboficiales, miembros del nivel ejecutivo y agentes, hombres y mujeres de todas

\footnotetext{
${ }^{10}$ A finales de 1994, el gobierno de Jaime Castro, con un préstamo de la banca francesa por US\$ 25 millones, el cual comprometió vigencia futuras, contrató la modernización del CAD. Este proyecto se comenzó a ejecutar a partir de 1995 y en los años siguientes el sistema integrado de radio, voz, datos y vídeo no funcionó como se esperaba.
} 
las áreas de servicio. Las temáticas abordadas han sido: derecho de policía, derechos humanos, derecho internacional humanitario, gestión gerencial, gestión pública y seguridad y convivencia ciudadana, abuso sexual de menores, policía comunitaria, inglés, secretariado ejecutivo, informática, estandarización de procedimientos, manejo de la escena del delito, pedagogía y metodología de educación de adultos.

d) Con el objetivo de vincular a las comunidades al tema de inseguridad, el Comando de la Policía Metropolitana puso en marcha distintos programas especiales como las Escuelas de Seguridad Ciudadana, donde se capacita a la comunidad en temas de seguridad y convivencia, con el objetivo de mejorar los comportamientos ciudadanos y que los líderes capacitados orienten a sus comunidades y para que apoyen a las autoridades en la prevención de la violencia y de la delincuencia. A través de este mecanismo, hoy día la ciudad cuenta con más de 26.000 líderes formados.

Así mismo y en concordancia con lo anterior, impulsó en 1995 la creación de los Frentes Locales de Seguridad, que son organizaciones de carácter comunitario, que integran los vecinos por cuadras, sectores, barrios, conjuntos cerrados y edificios, con los cuales se busca combatir el miedo, la apatía, la indiferencia y la falta de solidaridad frente a la acción de los violentos y delincuentes. Existen en la actualidad 6.663 Frentes.

e) Se puso en funcionamiento una nueva modalidad del servicio policial que se impulsó en 1999, la implementación de la Policía Comunitaria (policía de cercanía) y que hoy cuenta con 1.116 policías, cuyo objetivo es acercar al policía a la comunidad y propiciar una cultura de seguridad ciudadana en el barrio o sector asignado, a través de la integración de la administración local, la policía y la comunidad en procura del mejoramiento de la calidad de vida. Según un estudio realizado por el Instituto de Desarrollo Humano de la Pontificia Universidad Javeriana (5), el 96,4\% de los encuestados manifestó que la Policía Comunitaria es una alternativa eficaz para reducir los delitos y mejorar la convivencia ciudadana, entre otros aspectos porque desarrolla procesos de concientización en la comunidad; genera compromiso, diálogo y confianza y trabaja en la prevención y reducción del delito.

f) La más reciente estrategia (año 2001) ha sido maximizar el número de policías en las calles, lo cual se logró con su disminución en cargos administrativos y su asignación a la vigilancia en calle. En la actualidad, cerca de 1.500 policías que estaban en el área administrativa y en algunos servicios especiales (escoltas, vigilando instalaciones o de apoyo a otras instituciones) han sido asignados a la vigilancia y seguridad ciudadana.

g) Con el apoyo de la Cámara de Comercio de Bogotá, desde el año 2001, se comenzó el programa de Zonas Seguras, en el cual se definieron 12 cuadrantes de la ciudad, especialmente áreas comerciales y de alta presencia ciudadana y donde la policía, con la participación y apoyo de la comunidad organizada hace presencia permanente. Además de la policía en carros y motos, cada zona cuenta con una unidad móvil que atiende las denuncias de los ciudadanos.

Este programa es un modelo de gestión de seguridad del espacio público, con zonas específicas de intervención denominadas 'cuadrantes', producto de un esfuerzo conjunto de las autoridades distritales, la Policía Metropolitana y el sector privado (Cámara de Comercio de Bogotá y la comunidad). Este programa está inspirado en el esquema de 'equipos de barrio' que utiliza la Policía de Proximidad y Comunitaria de España. Opera todos los días de 8:00 a.m. a 8:00 p.m. en las once zonas de la ciudad en las que se registra una mayor actividad comercial, educativa, recreativa y una alta concentración de población y que, por ello, necesita una atención especial por parte de las autoridades distritales y de la Policía.

Entre los objetivos fundamentales del programa se encuentran:

- fomentar los vínculos de solidaridad y confianza entre los ciudadanos y las autoridades,

mejorar los niveles de percepción y la 
sensación de los ciudadanos en materia de seguridad ciudadana, $y$

- fomentar la participación ciudadana mediante los Frentes de Seguridad.

En las zonas seguras, la Policía Metropolitana ha asignado más de 80 agentes profesionales, de su personal más preparado, y once bachilleres de Policía, quienes hacen presencia permanente en las once zonas, se encargan de tener una mayor cercanía con la ciudadanía y trabajan conjuntamente con los Frentes Locales de Seguridad para poner freno a la comisión de delitos.

En materia de acercamiento a la justicia, la Cámara de Comercio entregó 11 Unidades Móviles de Denuncia y Contravenciones, a través de las cuales los ciudadanos pueden denunciar en forma permanente todo hecho delictivo que ocurra en la zona y les permitirá a los policías judicializar casos de contravenciones. Además, a través de estas Unidades se están divulgando normas de convivencia y se realizan jornadas de capacitación para dar a conocer el Código de Policía a la ciudadanía. Las Unidades operan en remolques ubicados en sitios estratégicos de cada zona y están equipadas con los equipos necesarios para la recepción de las denuncias.

Para fortalecer estos mecanismos de acercamiento a la justicia, la Alcaldía Mayor, a través de la Secretaría de Gobierno, destinó 11 inspectores de Policía o asesores jurídicos que brindan atención permanente a la ciudadanía.

h) Finalmente, hay que resaltar la asignación a la Policía Metropolitana del manejo del tránsito en 1995, que no sólo ha contribuido a la disminución de las muertes en accidentes de tránsito, que pasaron de 1.352 en 1995 a 745 en 2001, sino que ha conllevado el mejoramiento de la seguridad en las calles, pues no se limitan a ser reguladores de la movilidad de vehículos y pasajeros sino que, como autoridad de policía, desempeñan igualmente funciones preventivas y represivas en el control de los delitos.

\section{Justicia cercana al ciudadano}

En esta línea se inscriben los programas de justicia alternativa como la resolución pacífica de conflictos entre particulares y en los hogares, sino también al fortalecimiento de la justicia punitiva, como es el caso de facilitar el acceso de la ciudadanía a los servicios que prestan la Fiscalía General de la Nación, el Instituto Nacional de Medicina Legal y la Policía Judicial.

Los conflictos generados en la ciudad como consecuencia de la intolerancia, los problemas al interior de los hogares, entre vecinos y en general de la violencia menor, han sido atendidos en las fortalecidas Comisarías de Familia, que pasaron de cinco en 1995 a 20 en el año 2001, con la creación de doce Unidades de Mediación y Conciliación y dos Casas de Justicia que, aparte de atender directamente este tipo de problemas en las localidades, han propiciado la capacitación de, aproximadamente, 1.700 líderes que se han convertido en mediadores comunitarios que atienden casos diversos ${ }^{11}$.

En lo que tiene que ver con el fortalecimiento de la justicia punitiva, se creó la Unidad Permanente de Justicia, de la cual hacen parte la Fiscalía General de la Nación, el Instituto Nacional de Medicina Legal, la Policía Metropolitana y de

\footnotetext{
11 Dos casos atendidos por mediadores comunitarios: 1. Conflicto entre una hija de 14 años y los padres. La hija se había tornado bastante rebelde, no colaboraba en la casa. El mediador invita a los padres y a la niña, quien manifiesta que siente que está creciendo y que ellos la están maltratando; siente que la comunicación es "a los gritos" y que, igual, a ella eso le da mal genio. Se clarifica la comunicación de las dos partes. Se firma un acuerdo en el que se piden perdón mutuamente y los padres se comprometen a ser menos agresivos y ella se compromete a colaborar en las tareas del hogar y ellos le colaborarán en las tareas del colegio. Se le ha hecho seguimiento al caso y ha mejorado la situación de la familia.

2. Un muchacho es adicto a los juegos y la esposa ve cómo el hogar se va deteriorando, dado que él ya no permanece en la casa. Ella sabe que no le es infiel y que la sigue queriendo pero ha abandonado las actividades comunes. En la mediación ella le hace saber que ha cambiado, él identifica el problema y siente que no lo puede controlar y se compromete a empezar a cambiar algunas actitudes y algunos manejos de tiempo. Manifiesta que quiere a la esposa pero identifica su problema de adicción. El mediador consigue algunos materiales que le ayudan a identificar y clarificar su adicción, lo cual posibilitó, en dos o tres sesiones tener mayores claridades sobre el problema y le permitió buscar ayuda profesional. El caso ha avanzado en varias sesiones con una gran mejoría.
} 
Tránsito y una Inspección de Policía, la cual atiende las 24 horas del día. En ella, la Fiscalía y Medicina Legal definen en menos de dos días la situación de personas sindicadas de delitos. En la Unidad Permanente de Justicia, en los dos últimos años se ha logrado poner a disposición de la justicia más de 20.000 personas que habían cometido delitos. Igualmente, existen espacios en donde en los últimos dos años se han sancionado con retención, de no más de 36 horas, a más de 120.000 infractores, a quienes se les han decomisado armas de fuego y cortopunzantes.

Cuando no se contaba con esta Unidad, la Policía, en la mayoría de los casos, tenía que dejar libre a los infractores y la máxima sanción era el decomiso de los elementos y un llamado de atención verbal ya que no existían espacios para la retención temporal. En el caso de los delincuentes 0 de las personas que cometían delitos, la Fiscalía se demoraba varias semanas en definir la situación de los sindicados; hoy lo hace en dos días.

En esta misma línea, se ha ampliado la capacidad de la Cárcel Distrital de 450 cupos a 1.100, en una moderna edificación en la que se ha implementado un programa de resocialización que busca dignificar al recluso y fortalecer su autoestima. Con estos programas se ha logrado reducir la violencia y el consumo de drogas con buenos resultados, como quiera que en los últimos seis años se ha presentado una sola muerte violenta (suicidio), mientras que en el 2000 en las otras dos cárceles de la ciudad, La Picota y La Modelo, sumaron más de 130 homicidios comunes.

\section{Atención a jóvenes involucrados en asuntos de violencia y consumo de drogas}

Desde 1998, la administración distrital diseñó un proyecto orientado a reducir los factores asociados con la violencia juvenil, por medio del cual se ha vinculado a más de 20.000 jóvenes. Los componentes del programa son: educación, ocupación del tiempo libre, alternativas para generación de ingresos por actividades legales y la participación juvenil. Se han desarrollado las siguientes actividades: bachilleratos cortos con énfasis en convivencia para jóvenes pertenecientes a bandas y pandillas en proceso de reinserción, formación para el trabajo, desarrollo de hábitos y competencias básicas, actividades culturales, recreativas y de formación para el manejo del conflicto en instituciones escolares.

Finalmente, se apoyan iniciativas juveniles para la convivencia, a través de concursos como Los jóvenes conviven por Bogotá, entre las cuales se destacan los proyectos que buscan reinsertar jóvenes a procesos educativos, laborales y sociales.

\section{Mejoramiento de la convivencia y recuperación de sitios críticos}

A partir de la teoría de las ventanas rotas de Kelling y Coles (1997), en los últimos tres años, el Programa Misión Bogotá centró su intervención en la recuperación de espacios críticos en materia de seguridad y convivencia, para lo cual, con el apoyo de la Policía Metropolitana, definió y aplicó planes y acciones tendientes a mejorar la seguridad y fortalecer los lazos de afiliación que vinculan a los ciudadanos con sus entornos comunitarios.

Igualmente, con el apoyo de la Policía Comunitaria se constituyó en el generador de espacios de inclusión social para sectores de la población que habían sido vistos desde las políticas como receptores de servicios y no como agentes de desarrollo, como trabajadoras sexuales y habitantes de la calle (indigentes), algunos de los cuales hoy hacen parte del programa 'Guías Cívicos'.

En esta misma línea, a través del programa Renovación Urbana, desde 1998 se intervino el sitio más violento de la ciudad conocido como El Cartucho, donde se expendía y comercializaban drogas y armas y se organizaban actividades delictivas. Este sitio, conocido en otras ciudades como 'olla', se había constituido en el lugar donde se desarrollaban actividades delincuenciales $\sin$ que las autoridades distritales y nacionales pudiesen hacer algo, hasta 1998 cuando se tomó la decisión de acabarlo de raíz para construir allí un parque.

La intervención de este sitio se ha realizado desde los ámbitos social, policivo y administrativo y los 
resultados han sido importantes en materia de reducción de los índices de violencia y delincuencia de la ciudad y de reinserción social a los ciudadanos que habitan esta zona tales como:

- resocialización y traslado de negocios de acopio y comercialización de material reciclable, proceso coordinado por el DAMA pero que recoge el esfuerzo de diversas entidades del Distrito.

- Plan de Gestión Social que favoreció la formulación e implementación de una serie de proyectos y estrategias interinstitucionales que permitieron la atención de diversos grupos poblacionales asentados en la zona. La coordinación estuvo bajo la tutela del DABS.

\section{Recuperación del espacio público}

La recuperación del espacio público, no sólo contribuye a mejorar la percepción de seguridad, sino que impacta el medio ambiente y se desconfigura la triada delincuente, entorno y víctima, indispensable para que se posibilite el delito. Prueba de ello son las reducciones sustanciales de la actividad delictiva a lo largo de los últimos tres años en el centro de la ciudad y distintos lugares donde se llevó a cabo esta política. Entre 2001 y 2002, se recuperaron $553.372 \mathrm{~m}^{2}$ de espacio público.

\section{Hora zanahoria}

Se reafirma el efecto positivo que la medida de la 'hora zanahoria' tuvo en la reducción de los homicidios y otros delitos. La medida, aplicada a partir de diciembre de 1995, consiste en disminuir el tiempo de expendio de licor en los establecimientos autorizados para ello hasta la 1:00 a.m. y no hasta las 5:00 a.m. como estaba establecido. Como ya se mencionó, un estudio de la Universidad de los Andes atribuye a la 'hora zanahoria' una reducción del homicidio común del $8 \%$. Cabe anotar, que hasta el momento no se ha realizado un análisis detallado de los efectos de esta medida sobre la reducción de las muertes de tránsito. Sin embargo, como se puede observar en la figura de las muertes en accidentes de tránsito, es a partir de diciembre de 1995 y, en especial, 1996 cuando se registra una fuerte caída de este tipo de hechos en la ciudad.

\section{Desarme}

Al igual que la 'hora zanahoria', esta medida por sí sola no explica la reducción reportada para los homicidios y las lesiones comunes. Sólo basta recordar que a esta medida, el estudio de la Universidad de los Andes le atribuye una reducción del $14 \%$ en los homicidios comunes ocurridos los tres últimos años. De este programa también hay que destacar las acciones de entrega voluntaria de armas, el desarme de los colegios y la incautación y decomiso de armas legales e ilegales que han propiciado entre la ciudadanía espacios de reflexión sobre el uso de las armas a la hora de resolver los conflictos.

Por otro lado, adicional a las jornadas de desarme en los últimos años se incrementó el decomiso de armas, en especial de armas ilegales. Según reportes de la Policía Metropolitana, se pasó de algo más de 1.000 armas ilegales decomisadas en 1994 a más de 6.000 en el año 2001.

\section{Institucionalización de los temas de violencia y delincuencia}

La suma y continuidad de los programas, estrategias y acciones enumeradas anteriormente, han contribuido a la reducción de la violencia y de la delincuencia en la ciudad, junto con el hecho de que Bogotá ha logrado institucionalizar el tema, a partir de una consejería en 1995 hasta contar con una Subsecretaría para Asuntos de Convivencia y Seguridad Ciudadana en 1996 (figura 6 ). Hoy, un grupo de funcionarios públicos profesionales (un subsecretario, tres directores y 25 funcionarios), está dedicado a pensar y analizar esta problemática con el fin de fortalecer día a día la política pública que en esta materia se ha venido construyendo a lo largo de ocho años. Estos factores indudablemente apuntan a la comprensión del fenómeno delictivo en la ciudad y orientan su control.

\section{Manejo de indicadores de violencia y delincuencia e investigaciones}

Hoy la ciudad cuenta con el Sistema Unificado de Información de Violencia y Delincuencia, del cual hacen parte Medicina Legal, la Policía Metropolitana y la Alcaldía Mayor. Con base en esta información y otras fuentes, desde 1995, la 




Figura 6. Organigrama de la Subsecretaría de Asuntos de Convivencia y Seguridad Ciudadana de Bogotá, D.C.

administración distrital ha venido adelantando una serie de investigaciones sobre diferentes tópicos que afectan la seguridad y la convivencia. Es así como se han hecho investigaciones sobre percepción cuyos resultados se publicaron en el texto Territorios del miedo sobre violencia y jóvenes (Alonso Salazar); violencia homicida (Universidad de los Andes); delitos de mayor impacto (Universidades de los Andes y Universidad Nacional); violencia sexual (Universidad de los Andes); accidentalidad vial (Universidad Nacional); carrera delictiva (Universidad Nacional), entre otras ${ }^{12}$.

${ }^{12}$ Caracterización de la Violencia Homicida en Bogotá (2000), se contrató a la Universidad de Los Andes. Accidentalidad Vial en Bogotá (2000), se contrató a la Universidad Nacional de Colombia.

La violencia sexual en Bogotá (2000), se contrató a la Universidad de Los Andes.

Atraco Callejero, Robo de Automotores, Hurto a Residencias y a Establecimientos Comerciales en Bogotá (2000), se contrató a la Universidad de Los Andes.

Caracterización de la seguridad y la convivencia en Bogotá: informe general (2000), se contrató un grupo de expertos en temas de seguridad para su realización. Interpretación socio jurídica del estado actual de los delitos menores en Santa Fe de Bogotá D.C. (2000), se contrató a la Universidad Nacional de Colombia.

Caracterización de las conductas suicidas en Bogotá D.C. (2001), se contrató a la Universidad Nacional de Colombia.

La carrera delincuencial en Bogotá D.C. (2001), se contrató a la Universidad Nacional de Colombia.

Opiniones, percepciones de los jóvenes de Santa Fe de Bogotá (1999).

Estudio sobre las distintas causas de violencia intrafamiliar reportadas en el último año por las Comisarías de Familia del Distrito Capital (2000).

Las emisoras escolares como herramienta en la gestión del conflicto y la promoción de la convivencia (2001).
Con base en el comportamiento de las cifras y los resultados de estas investigaciones, a través del Consejo Distrital de Seguridad ${ }^{13}$ y el Comité de Vigilancia Epidemiológica ${ }^{14}$, se diseñan estrategias de intervención y se hace seguimiento a las acciones definidas para su evaluación.

\section{Fortalecimiento de la investigación criminal}

En el campo de la investigación criminal, junto con el Departamento Administrativo de Seguridad, la Fiscalía General de la Nación, Medicina Legal, la SIJIN y la DIJIN de la Policía Nacional, en la actualidad, se actualizan los conocimientos de

\footnotetext{
${ }_{13}$ Compuesto por el alcalde mayor, el Comandante de la XIII Brigada, el comandante de la Policía Metropolitana, el subdirector del Departamento Administrativo de Seguridad, el delegado del Procurador General de la Nación, el director seccional de Orden Público y la Secretaria de Gobierno del Distrito Capital. Este Consejo tiene, entre otras funciones, diseñar políticas e intervenciones para reducir la violencia y la delincuencia. (Decreto 2615 de 1991).

${ }^{14}$ A instancias de la Secretaría de Gobierno, desde 1995, todos los jueves de 7:00 a 9:00 a.m. se convoca a este comité, que como su nombre lo indica, se encarga de la planificación, ejecución y evaluación de programas para prevenir las lesiones de causa externa a partir del análisis e interpretación sistemática de los datos de violencia y delincuencia de Bogotá que entrega el Sistema Unificado de Información de Violencia y Delincuencia, SUIVD. Las entidades participantes, además de la Secretaría de Gobierno, son, el Instituto Nacional de Medicina Legal y Ciencias Forenses, la Policía Metropolitana, el Instituto Distrital de Cultura y Turismo, la Unidad Coordinadora de Prevención Integral, UCPI, el Instituto Colombiano de Bienestar Familiar ICBF, la Unidad de Prevención y Atención de Emergencias, la Secretaría de Tránsito, el Departamento Administrativo de Seguridad, DAS, e invitados especiales de acuerdo con las temáticas tratadas. (Decreto 649 de 1996).
} 
policía judicial y se estandarizan los procedimientos de policía para la inspección del lugar de los hechos delictivos buscando dar mayores elementos a los fiscales para iniciar una investigación y llevarla a buen término. Con estas instituciones se conformará el Comité de Política Criminal que busca diseñar y aplicar de manera interinstitucional la política criminal del Distrito.

\section{Conclusiones}

En los últimos años se ha producido un desplazamiento en la concepción del manejo de la seguridad en Bogotá, dándole un giro hacia un sentido más integral y enfocado en las personas. Por un lado, se ha precisado que las diferentes entidades del Distrito tienen un papel que cumplir en términos de la prevención y atención de la violencia y la delincuencia y en el incremento de la percepción de seguridad y que ésta no es labor exclusiva de la Policía y de los organismos de justicia. Por otro lado, la seguridad se ha enfocado a proveerle a los ciudadanos condiciones seguras, es decir, espacios públicos seguros, condiciones de denuncia amables, atención oportuna, etc.

La base teórica para este proceso se inicia y sustenta en el Programa de Cultura Ciudadana, desarrollado por el alcalde Antanas Mockus en su primera administración (1995-1997), en el que se plantea "el divorcio entre la regulación jurídica (lo legal), la regulación cultural (colectiva y variable según el contexto) y la regulación moral (individual), para explicar de manera precisa las dificultades claves de la convivencia" (6), y a partir de allí propuso la "interacción intensificada" de las tres regulaciones para orientar las actividades del Programa de Seguridad y Convivencia. En el Plan de Desarrollo 'Formar Ciudad', en 1995, se aprobaron cuatro objetivos relativos a este programa: "a) lograr un mayor cumplimiento de las normas de convivencia; b) dotar a algunos ciudadanos de una mayor capacidad para llevar a otros a cumplir las normas pacíficamente; c) mejorar la capacidad para concertar acuerdos y dar solución pacífica a los conflictos ciudadanos (expresión, interpretación) por medio del arte, actividades culturales, la recreación y el deporte".

Diversos programas y actividades se han venido realizando desde entonces en forma continua y sostenida por las administraciones siguientes y han adquirido la categoría de políticas públicas de convivencia y seguridad ciudadanas. Así, durante la administración de Enrique Peñalosa, aunque se dio continuidad al proceso, se hizo énfasis en el principio de autoridad, lo que se vio reflejado en la propuesta de recuperación del espacio público y mejoramiento de entornos urbanos críticos, entre otros aspectos.

La construcción de la política de seguridad en el Distrito ha hecho parte de un proceso de consolidación de esfuerzos interinstitucionales distritales y locales; asimismo, desde las directrices de la Secretaría de Gobierno, a través de la Subsecretaría de Asuntos de Convivencia y Seguridad Ciudadana, con un proceso continuado desde 1995, que le ha permitido a la ciudad afianzarse en una cultura de convivencia y seguridad. Los habitantes de Bogotá se sienten más identificados con ella y esto, en parte, por la garantía al respeto a su vida y bienes. Hay muchos proyectos y actividades que deben implementarse, crearse y mejorar los ya existentes dado que aún no estamos a un nivel que nos permita reclamar a Bogotá como una ciudad segura.

\section{Referencias}

1. Villaveces A, Cummings $\mathbf{P}$, Espitia VE, Koepsell TD, McKnight B, Kallermann AL. Effect of ban carrying ferearms on homicide rates in 2 Colombian cities. JAMA 2000;383:1205.

2. Beltrán I, Fernández AM, Gaitán F. Política de seguridad y gasto en Bogotá. Misión de la reforma institucional del Distrito. Bogotá, Colombia: Universidad del Rosario; 2000.

3. Deas M, Gaitán F. Dos ensayos especulativos sobre la violencia en Colombia. Bogotá, Colombia: Fonade, Departamento Nacional de Planeación; 1995.

4. Cámara de Comercio de Bogotá, Misión Siglo XXI. Estudio prospectivo de seguridad, Bogotá, 1996. Bogotá: Cámara de Comercio; 1996. p. 56.

5. Pontifica Universidad Javeriana, Facultad de Educación, Instituto de Desarrollo Humano.Informe de Consultoría. Percepción y expectativas ciudadanas sobre la modalidad de Policía Comunitaria en Santa Fe de Bogotá. Bogotá: Pontifica Universidad Javeriana, Facultad de Educación, Instituto de Desarrollo Humano; 2002.

6. Mockus A. Cultura ciudadana, programa contra la violencia en Santa Fe de Bogotá, Colombia, 1995-1997. Washington, D.C.: BID; 2001; http://www.iadb.org. 